\title{
Digitale Zentralbankwährungen: Chancen, Risiken und Blockchain-Technologie
}

\author{
Zahlreiche Zentralbanken planen innerhalb der nächsten Jahre, eigene digitale \\ Zentralbankwährungen einzuführen. Die Blockchain-Technologie kann dafür die \\ technologische Basis darstellen. Zentralbanken experimentieren mit dieser Technologie, um \\ Währungen perspektivisch auf Blockchain-Basis abzubilden. Mögliche Vorteile einer digitalen \\ Zentralbankwährung wären eine höhere finanzielle Stabilität, eine höhere Sicherheit und \\ Effizienz im Zahlungsverkehr und ein höherer Automatisierungsgrad von Geschäftsprozessen. \\ Risiken beständen darin, dass es nach einer Einführung zu einem digitalen Bank Run \\ kommen könnte, dass Banken immens an Bedeutung verlieren könnten und letztlich die \\ Datenschutzproblematik geklärt werden muss.
}

2008 war ein besonderes Jahr für das globale Finanzsystem. Neben dem Beginn der Finanzkrise wurde in diesem Jahr eine fundamental neue Form des Geldes geboren: Das Aufkommen von Bitcoin markierte den Start für sogenannte Kryptowerte - auf einer Distributed-LedgerTechnologie (DLT) und Kryptografie basiertes, digitales Geld. Die Geburt dieser Kryptowerte und spätestens die Ankündigung von Facebook, im Sommer 2019 mit „Libra“ einen globalen DLT-basierten Kryptowert als weltweites Zahlungsmittel etablieren zu wollen, haben Zentralbanken weltweit dazu veranlasst, sich mit der Digitalisierung des Geldes und der Emission eigener digitaler Zentralbankwährungen (Central Bank Digital Currencies, CBDC) auseinanderzusetzen (Groß et al., 2019).

Eine Anfang des Jahres veröffentlichte Studie der Bank für Internationalen Zahlungsausgleich (BIZ) zeigt, dass aktuell $70 \%$ aller weltweiten Zentralbanken die Ausgabe einer eigenen digitalen Zentralbankwährung untersuchen (Boar et al., 2020). $10 \%$ dieser Zentralbanken planen innerhalb der nächsten ein bis drei Jahre, eine solche digitale Währung einzuführen - in den nächsten sechs Jahren sogar $20 \%$. Es zeigt sich insofern ein klarer Trend: Immer mehr Zentralbanken beschäftigen sich intensiv mit dem Thema CBDC und es ist nur eine Frage der Zeit, bis die ersten CBDC auf den Markt kommen.

CBDC lassen sich in Retail- und Wholesale-CBDC unterteilen. Bei Wholesale-CBDC ist der Zugang auf spe-

(c) Der/die Autor(en) 2020. Open Access: Dieser Artikel wird unter der Creative Commons Namensnennung 4.0 International Lizenz (https:// creativecommons.org/licenses/by/4.0/deed.de) veröffentlicht.

Open Access wird durch die ZBW - Leibniz-Informationszentrum Wirtschaft gefördert. zielle Finanzinstitutionen (vor allem Banken) beschränkt. Dementsprechend würden Wholesale-CBDC vor allem im Interbankenmarkt Anwendung finden und könnten nicht als allgemeines Zahlungsmittel genutzt werden. RetailCBDC hingegen sind der breiten Öffentlichkeit zugänglich - hier geht es letztendlich darum, physisches Bargeld zu digitalisieren. Während Wholesale-CBDC durch die alleinige Anwendung im Interbankenmarkt keine fundamentalen Veränderungen des zweigliedrigen Geldsystems aus Zentral- und Geschäftsbanken hervorrufen würden, wäre die Einführung von Retail-CBDC als allgemeines Zahlungsmittel eine erhebliche Veränderung des bestehenden Geldsystems. Insofern liegt der Fokus des vorliegenden Beitrags auf Retail-CBDC.

Aktuell gibt es international viele CBDC-Projekte. China hat im April 2020 als erste entwickelte Volkswirtschaft einen Testlauf des CBDC-Projekts DC/EP gestartet. Weitere Tests sollen zu den Olympischen Winterspielen 2022

Jonas Groß, M. Sc., ist wissenschaftlicher Mitarbeiter am Frankfurt School Blockchain Center und der Universität Bayreuth.

Manuel Klein ist Consultant bei FactSet und Vorstandsmitglied im Verein Monetative e.V.

Prof. Dr. Philipp Sandner ist Leiter des Frankfurt School Blockchain Center. 
durchgeführt werden. Die Schwedische Zentralbank analysiert bereits seit 2017 die Ausgabe einer digitalen Variante der Schwedischen Krona (E-Krona) und testet einen DLT-basierten E-Krona-Prototypen (Riksbank, 2020). In der Karibik untersucht die Eastern Caribbean Central Bank die Anwendbarkeit der DLT für einen digitalen Eastern Caribbean Dollar. Der Sand-Dollar der Bahamas verfolgt ein ähnliches Ziel und ist seit Dezember 2019 in einer Pilot-Phase für die Bürger der Bahamas verfügbar. Auch der SOV, ein Kryptowert der Marshall Islands, soll in den nächsten Monaten getestet werden. Vorreiter der theoretischen Debatte rund um CBDC war die Bank of England, die 2015 das Thema aufgrund der starken Dynamik um Kryptowerte und DLT in ihre Research Agenda aufgenommen hat.

\section{CBDC und das aktuelle Geldsystem}

Um die ökonomischen Implikationen von CBDC einordnen zu können, lohnt es sich, CBDC mit Hinblick auf den Aufbau des bestehenden Geldsystems zu diskutieren. Derzeit existieren drei Formen von umlaufendem Geld:

- Bargeld stellt den Anker des bestehenden Geldsystems dar. Es ist das einzige gesetzliche Zahlungsmittel und kann nur von der Zentralbank emittiert werden. Es ist Peer-to-Peer, also ohne Intermediär, transferierbar, ist nicht digital, jedoch der breiten Öffentlichkeit zugänglich. Zudem ermöglicht es anonyme Transaktionen und ist nicht verzinst.

- Giralgeld wird von Geschäftsbanken proaktiv emittiert und stellt einen Anspruch an eine Geschäftsbank auf Auszahlung von Bargeld dar. Der Kontostand eines Bankkunden bedeutet insofern, dass Bargeld in Höhe des angezeigten Betrags vom Kunden von seinem Bankkonto abgehoben werden kann. Das Giralgeld im Euroraum ist laut Europäischer Zentralbank (EZB) jedoch nur durch rund $13 \%$ Bargeld und sonst vor allem durch Forderungen an Kreditnehmer und andere Vermögenswerte gedeckt (EZB Statistical Data Warehouse, 2020). Dies macht das Halten von Giralgeld risikobehaftet: Bankkunden sind Liquiditäts-, Markt- und Gläubigerrisiken der Kreditnehmer ausgesetzt. Trotzdem ist Giralgeld 1:1 an den Wert des Bargelds gebunden und kann zum selben Nennwert in bar abgehoben werden. Der Zinssatz auf Giralgeld-Einlagen wird von der jeweiligen Bank festgelegt, orientiert sich allerdings an den Refinanzierungszinsätzen der Banken bei der Zentralbank.

- Zentralbankreserven (Reserven) werden von der Zentralbank emittiert und sind der breiten Öffentlichkeit nicht zugänglich. Es handelt sich um von der Zentral- bank ausgegebenes Geld, sodass der Halter des Geldes - anders als beim Giralgeld der Banken - keinem Liquiditäts- oder Gläubigerrisiko ausgesetzt ist. Zentralbanken können im Gegensatz zu privaten Banken systembedingt und per Definition nicht insolvent gehen, da sie die einzigen Institutionen sind, die gesetzliche Zahlungsmittel erzeugen und im Notfall auch mit negativem Eigenkapital arbeiten können (Bunea et al., 2016). Somit stellen Zentralbankreserven (wie auch Bargeld) ein risikofreies Geld dar - sie sind allerdings im Gegensatz zu Bargeld über digitale Zahlungssysteme übertragbar. Reserven sind zinstragend ausgestaltet und werden zum Einlagezinssatz der Zentralbank verzinst.

Retail-CBDC würden eine weitere, vierte Geldart darstellen, die letztlich das von der Zentralbank ausgegebene Bargeld digitalisieren würde. Eine Retail-CBDC würde parallel zum Giralgeld der Banken und Bargeld zirkulieren und als generelles Zahlungsmittel verwendet werden. CBDC lassen sich anhand zahlreicher Gestaltungsmerkmale unterscheiden (Hanl und Michaelis, 2019). Sie können Peerto-Peer handelbar sein (Wert-basierte CBDC) oder können über Konten bei der Zentralbank zugänglich gemacht werden (Konten-basierte CBDC). Eine Wert-basierte CBDC wäre dem aktuellen physischen Bargeld sehr ähnlich, da die CBDC-Einheiten als zwischen den Parteien direkt übertragbare Token in die Realwirtschaft emittiert werden würden. Somit wären CBDC wie Bargeld Peer-to-Peer, also ohne Intermediär, übertragbar. Eine Konten-basierte CBDC würde in der Übertragung Bankeinlagen ähneln.

Bei beiden Ausgestaltungsformen gibt es die Möglichkeit, dass die CBDC-Einheiten direkt von der Zentralbank ausgegeben werden oder dass Intermediäre, wie z. B. Banken, die digitalen Geldeinheiten von der Zentralbank erhalten und diese dann für den Kunden verwalten. Zudem kann eine CBDC - ähnlich wie Giralgeld - einen Zinsertrag abwerfen. Eine zinstragende CBDC hätte mitunter erhebliche Auswirkungen auf die Geldpolitik der Zentralbanken (Hanl und Michaelis, 2019). Im Falle einer Retail-CBDC würden die von der Zentralbank gesetzen Zinsen nicht wie im aktuellen System nur für die Banken, sondern für alle, also auch für Nicht-Banken, gelten. Dies könnte die Effizienz der Geldpolitik erhöhen, den Transmissionsmechanismus auf die Zinsen der Banken jedoch verändern. Einige Ökonomen sehen auch die Möglichkeit, durch CBDC effektiv Negativzinsen besser durchzusetzen.

\section{Motive einer CBDC-Einführung}

Die Motive, eine Retail-CBDC einzuführen, sind vielfältig (vgl. Tabelle 1, Boar et al., 2020; Bank of England, 2020). Zentralbanker erhoffen sich von einer CBDC-Einführung 
Tabelle 1

\section{Motive einer CBDC-Einführung}

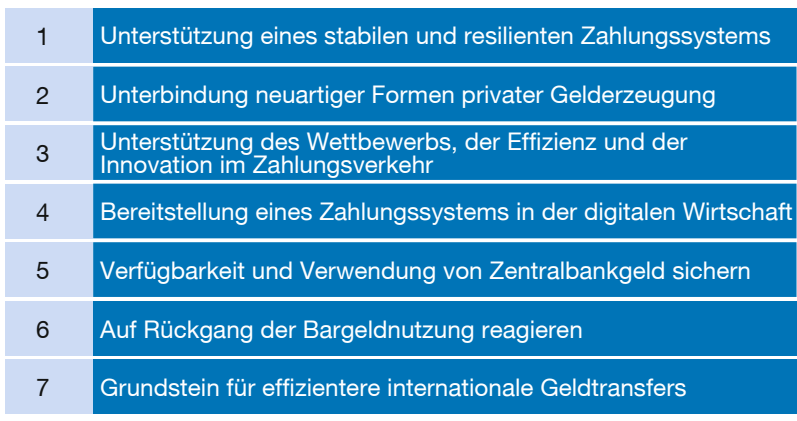

Quelle: Bank of England (2020).

primär eine erhöhte Resilienz des Finanzsystems, was mit einer höheren finanziellen Stabilität einhergehen würde: Zum einen würde eine CBDC-Einführung zu Geldflüssen vom Bankensektor zur Zentralbank führen. Dadurch würde das Ausmaß möglicher Bankturbulenzen auf die Volkswirtschaft reduziert werden. Zum anderen würde die operative Resilienz der Zahlungsnetzwerke verbessert, indem Zahlungsmöglichkeiten diversifiziert werden und ein sicheres und öffentliches digitales Zahlungssystem geschaffen wird. Somit wäre es in einer Welt mit CBDC deutlich unwahrscheinlicher als im aktuellen System, dass private Zahlungsnetzwerke, wie Mastercard, Visa oder auch ApplePay, und öffentliche Zahlungssysteme, z.B. von der Zentralbank, gleichzeitig ausfallen würden (Delivery vs. Payment).

Um die Sicherheit des Zahlungsverkehrs zu erhöhen, könnte eine DLT, wie die Blockchain-Technologie, als technologische Basis für eine CBDC eingesetzt werden. Im aktuellen Geldsystem werden Transaktionsdaten meist zentral auf den Servern der beteiligten (Zentral-) Banken gespeichert. Bei DLT-Systemen werden Daten hingegen gleichzeitig auf mehreren Computern und somit in einem verteilten Register gespeichert. Diese dezentrale Speicherung macht DLT-Systeme widerstandsfähiger gegen Hackerangriffe, da Single-Points-of-Failure entfallen. Aufgrund der Mehrfachspeicherung der Transaktionsdaten ist es nicht möglich, Transaktionsdaten zu manipulieren oder im Nachhinein anzupassen.

Außerdem könnten CBDC Marktversagen im aktuellen Zahlungsverkehrssystem adressieren. Zwar scheint es heutzutage der Fall zu sein, dass digitale Zahlungen z. B. per Kreditkarten, EC-Karten oder Smartphones bereits instantan ablaufen und verarbeitet werden - allerdings nimmt die Abwicklung (Settlement) der Zahlungen häufig mehrere Tage Zeit in Anspruch, sodass der Verkäufer mehrere Tage auf den Zahlungseingang warten muss.
Somit gibt es mehrere Parteien, die in Vorauszahlung treten und einem Gläubigerrisiko ausgesetzt sind (Bank of England, 2020). Eine CBDC könnte die Abwicklung der Zahlungen beschleunigen und die Effizienz steigern. Im Kontext des Settlements wäre die Nutzung einer DLT von Vorteil, da nur DLT-Systeme es ermöglichen, Leistung (z. B. Kauf eines Guts) und Gegenleistung (z. B. die Bezahlung des Guts) auf integrierten Plattformen zu organisieren, was neben Real-Time-Settlements auch zu automatisierter Leistungserbringung führen würde.

Zudem könnte eine CBDC dabei helfen, Transaktionskosten zu senken. Vor allem im grenzüberschreitenden Zahlungsverkehr sind Transaktionskosten aktuell noch sehr hoch - im Durchschnitt kostet eine grenzüberschreitende Transaktion $7 \%$ Gebühren und Auslandsüberweisungen dauern teilweise bis zu zehn Tage (o. V., Handelsblatt, 2019). Hier könnte ein DLT-basiertes System ansetzen und die Komplexität der Zahlungssysteme reduzieren, da viele Mittelsmänner in DLT-Systemen nicht mehr benötigt werden würden (z. B. Clearing-Stellen). Dies würde zu erheblichen Kostensenkungen und einer schnelleren Abwicklung von Transaktionen führen.

Die Einführung einer DLT-basierten CBDC würde zudem Geldflüsse programmierbar machen. Bei programmierbaren Transaktionen handelt es sich um Zahlungsvorgänge, die einer bestimmten Logik folgen und auch automatisch ausgeführt werden können. Derzeit gibt es etwa bereits Daueraufträge, Treuhandprozesse, Zinszahlungen, LeasingZahlungen und Factoring-Modelle, die letztlich programmierte Transaktionen sind, bei denen Zahlungen immer dann stattfinden, wenn bestimmte Bedingungen erfüllt sind bzw. ein bestimmtes Ereignis eingetreten ist. Mittels DLT können solche und weitaus komplexere Prozesse mit wenigen Zeilen Programmcode durch „Smart Contracts“ und ohne Intermediäre umgesetzt und automatisiert werden.

Programmierbare Transaktionen haben vor allem im Kontext der Machine Economy enormes Potenzial. So würden es Smart Contracts ermöglichen, dass an die DLT angeschlossene Internet-of-Things (IoT)-Geräte, wie etwa Maschinen, Autos und Sensoren, Dienstleistungen auf Pay-Per-Use-Basis oder auch Leasing oder Factoring anbieten können. IoT Analytics erwartet, dass 2025 mehr als 20 Mrd. Geräte mit dem Internet verbunden sein werden. Die DLT ist am besten dafür geeignet, Millionen von Geräten mit einer eigenen digitalen Brieftasche (Wallet) auszustatten, über die dann CBDC direkt von Wallet zu Wallet transferiert werden können. Zudem könnten durch programmierbare Transaktionen z. B. Steuerzahlungen an das Finanzamt oder Dividendenzahlungen an Aktionäre automatisiert werden (Bank of England, 2020). Schließlich könnte eine DLT-basierte CBDC Mikrozahlungen ermög- 
lichen, da mithilfe der DLT kleine Transaktionen zu sehr geringen Transaktionskosten durchgeführt werden könnten. Dadurch könnte sich das Geschäftsvolumen von Mikrozahlungen deutlich erhöhen und es könnten sich neue Dienstleistungen rund um Mikrozahlungen entwickeln.

\section{Risiken einer CBDC-Einführung}

Aktuell diskutieren Zentralbanker und andere Ökonomen intensiv darüber, wie genau ein Retail-CBDC-System ausgestaltet werden sollte, ohne die finanzielle Stabilität des bestehenden Geldsystems zu gefährden. Bundesbankpräsident Jens Weidmann verweist auf das Risiko eines „digitalen Bank Runs“, wenn Kunden ihre Bank-Einlagen in das neue digitale Zentralbankgeld ,per Mausklick“ umtauschen könnten (o.V., Handelsblatt, 2020). Zwar kann es auch im aktuellen Geldsystem zu Bank Runs kommen, allerdings sind solche Runs durch Friktionen und Kosten für das Abheben und Lagern von physischem Bargeld beschränkt. Diese Friktionen und Kosten wären bei CBDC deutlich geringer, da Lagerkosten und Versorgungsengpässe an Geldautomaten entfallen würden (Bank of England, 2020).

Würde es zu einem digitalen Bank Run kommen, könnten die Liquiditätsbestände der Banken in Form von Zentralbankreserven durch den Abfluss von Einlagen aus dem Bankensystem zur Zentralbank (Disintermediation des Bankensektors) bedroht werden - im schlimmsten Fall könnten Bank-Insolvenzen drohen. Im Extremfall könnten Banken zu reinen Intermediären werden und keine Geldschöpfung per Kreditvergabe mehr betreiben. Allerdings ist eine gewisse Umschichtung der Kunden von Giralgeld in CBDC notwendig, damit CBDC letztlich für Transaktionen genutzt werden können. Es soll insofern gewährleistet werden, dass die Disintermediation nicht so groß ausfällt, dass Banken in finanzielle Schieflage geraten.

Bisher wurden verschiedene Konzepte entwickelt, wie ein digitaler Bank Run und eine Disintermediation des Bankensektors verhindert werden könnten:

- Autoren der Bank of England schlagen vor, ein paralleles Zahlungssystem der Zentralbank zu etablieren, das einen direkten Umtausch und digitales Abheben von Giralgeld in CBDC unmöglich macht (Kumhof und Noone, 2018).

- In einer Publikation der EZB wird ein zweistufiges (Tiered-)CBDC-System mit zwei unterschiedlichen Zinssätzen auf die vom Nicht-Bankensektor gehaltenen CBDC-Guthaben vorgeschlagen, um Banken-Disintermediation zu verhindern (Bindseil, 2020). Wird ein bestimmter CBDC-Schwellenwert auf dem Zentralbankkonto überschritten, würde der überschüssige CBDC-
Betrag mit $0 \%$ oder sogar negativ verzinst werden. Das macht das Halten von CBDC in großen Mengen also als Wertaufbewahrungsmittel - unattraktiv, da der überschüssige CBDC-Betrag niemals positiv verzinst werden würde. ${ }^{1}$

- Eine weitere Alternative wäre die Einführung von Maximalbeträgen, die Kunden in CBDC halten dürfen (Bank of England, 2020). Maximalbeträge würden ebenfalls dazu führen, dass CBDC primär für Transaktions- und nicht für Wertaufbewahrungszwecke genutzt würden. Dies würde das Geschäft der Banken schützen und somit eine erhebliche Disintermediation des Bankensektors verhindern.

Neben Gefahren für die finanzielle Stabilität werden häufig auch Risiken für den Datenschutz diskutiert. Heutzutage unterscheiden sich Transaktionen per Apple Pay, Paypal, per Banküberweisung oder per Bargeld anhand ihres Grads an Datensicherheit und Anonymität. Wird eine Zahlung z. B. über Apples Bezahldienst Apple Pay oder über Paypal abgewickelt, sind die (sensiblen) Transaktionsdaten für Apple oder Paypal einsehbar. Bei einer Banküberweisung können Banken auf die Transaktionsdetails zugreifen. Bargeldtransaktionen laufen jedoch auf Peer-toPeer-Basis ab und bieten, wenn gewünscht, Anonymität zwischen den Transaktionspartnern, aber vor allem auch gegenüber der Zentralbank. Ein hoher Grad an Datensicherheit und ein gewisses Maß an Anonymität sollte auch bei Transaktionen in CBDC gewährleistet werden.

Die aktuellen Forschungsbeiträge der EZB und der Bank of England verdeutlichen, dass es auch in CBDC-Systemen die Möglichkeit geben sollte, Transaktionen teilweise anonym durchzuführen. In einem im Dezember 2019 veröffentlichten Papier stellt die EZB (2020), ein konkretes DLT-basiertes Retail-CBDC-System vor, in dem (teilweise) anonyme Zahlungen gewährleistet werden. Die EZB emittiert in diesem System lediglich CBDC-Einheiten Banken übernehmen administrative Aufgaben, wie die Überprüfung von Anti-Geldwäsche-Bestimmungen und die Verwahrung der CBDC mithilfe eigener Wallets.

Innerhalb des Systems sind (teilweise) anonyme Zahlungen garantiert, die gleichzeitig Anti-Geldwäsche-Bestimmungen berücksichtigen. Technisch wird diese (Teil-)Anonymität

1 Die Idee einer zweistufigen CBDC-Verzinsung von Zentralbankguthaben ist nicht neu: Gegenwärtig ist das Sechsfache der Mindestreserve, die die Geschäftsbanken als Mindestanforderung an Liquidität bei der Zentralbank halten müssen, vom negativen Einlagezins ausgenommen, vgl. Deutsche Bundesbank (2020). In der Eurozone beträgt die Mindestreserve aktuell $1 \%$ der Bankeinlagen. Auf die überschüssigen Reserven, die Banken bei der Zentralbank halten, muss aktuell der Einlagezins in Höhe von $-0,5 \%$ gezahlt werden. 
dadurch umgesetzt, dass die Identität des Nutzers und die Transaktionshistorie der Zentralbank (und der Anti-Geldwäsche-Behörde) nicht bekannt gegeben werden, wenn sogenannte Anonymity Vouchers der Zahlung beigefügt werden. Jedem Bürger wird eine bestimmte Zahl an Anonymity Vouchers gutgeschrieben, mit denen anonyme Transaktionen durchgeführt werden können. Sobald alle Vouchers eingelöst worden sind, werden Zahlungen nicht mehr anonym durchgeführt. Ein Nachteil dieses konkreten von der EZB vorgeschlagenen Systems ist jedoch, dass die Zahlungsdetails für die abwickelnden Banken einsehbar sind. Die Anonymität ist insofern nur zwischen den Akteuren der Transaktion und der Zentralbank gegeben. Diese Einschränkung ist ein klarer Nachteil des Prototyps und sollte in zukünftigen Entwicklungen berücksichtigt werden. Dennoch ist dieses Paper ein guter Ausgangspunkt, um die Anonymität in Retail-CBDC im Kontext mit DLT weiter zu analysieren.

Auch die Bank of England betont in einem aktuellen Forschungspapier, dass ein CBDC-System mit Datenschutzbestimmungen, wie der Datenschutzgrundverordnung (DSGVO), in Einklang stehen muss. Es soll jedem Kunden das Recht eingeräumt werden, Kontrolle darüber zu erhalten, wie seine Daten genutzt werden und mit welchen Teilnehmern diese geteilt werden. Die Bank of England bringt die Option ins Spiel, dass Transaktionsdaten mit relevanten Behörden, die z.B. die Bekämpfung von Geldwäsche und Terrorfinanzierung adressieren, geteilt werden, allerdings nicht mit Verkäufern oder anderen Unternehmern, die die Kaufgewohnheiten der Kunden auswerten. Die beiden Vorschläge der EZB und der Bank of England weisen darauf hin, dass auch in einer CBDC-Welt Datenschutz einen hohen Stellenwert bekommen soll, was für eine weitreichende Verbreitung von CBDC essenziell ist.

\section{EZB intensiviert Bemühungen}

Es kann erwartet werden, dass die EZB weitere Ressourcen in die Erforschung von CBDC stecken wird. Christine Lagarde, Präsidentin der EZB, hat im November 2019 angekündigt, die Bemühungen der EZB im Bereich CBDC weiter zu intensivieren und eine eigene EZB-interne CBDCTaskforce ins Leben zu rufen. Auch der CBDC-Austausch mit anderen Zentralbanken soll weiter verbessert werden - vor allem durch ein gemeinsames CBDC-Projekt mit der
Bank für Internationalen Zahlungsausgleich (BIZ). Dem Konsortium gehören unter anderem die Bank of England, Bank of Japan, die Riksbank und weitere Zentralbanken, an, um Erfahrungen aus ihren Analysen potenzieller CBDC-Use-Cases, und CBDC-Probleme auszutauschen und um die Implikationen einer CBDC gemeinsam zu untersuchen. Die Bemühungen der EZB zeigen, dass eine CBDC in der Eurozone durchaus Realität werden könnte - möglicherweise sogar auf DLT-Basis.

\section{Literatur}

Bank of England (2020), Central Bank Digital Currency - Opportunities, Challenges and Design, Bank of England Discussion Paper, https:// www.bankofengland.co.uk/paper/2020/central-bank-digital-currency-opportunities-challenges-and-design-discussion-paper (18. März 2020).

Bindseil, U. (2020), Tiered CBDC and the Financial System", ECB Working Paper, 2351.

Boar, D., H. Holden und A. Wadsworth (2020), Impending Arrival - a Sequel to the Survey on Central Bank Digital Currency, BIS Papers, 107.

Bunea, D., P. Karakitsos, N. Merriman und W. Studener (2016), Profit Distribution and Loss Coverage Rules for Central Banks, European Central Bank - Occasional Paper Series, 169.

Deutsche Bundesbank (2020), Zweistufiges System für die Verzinsung von Überschussreserven, https://www.bundesbank.de/de/aufgaben/ geldpolitik/ueberschussreserven (20. März 2020).

EZB (2020), Exploring Anonymity in Central Bank Digital Currencies, https://www.ecb.europa.eu/paym/intro/publications/pdf/ecb.mipinfocus191217.en.pdf (6. März 2020).

EZB Statistical Data Warehouse (2020), Monetary Statistics January 2020, http://sdw.ecb.europa.eu/reports.do?node=1000003478 (20. März 2020).

Groß, J., B. Herz und J. Schiller (2019), Libra - Konzept und wirtschaftspolitische Implikationen, Wirtschaftsdienst, 99(9), 625-631, https:// www.wirtschaftsdienst.eu/inhalt/jahr/2019/heft/9/beitrag/libra-konzept-und-wirtschaftspolitische-implikationen.html.

Hanl, A. und J. Michaelis (2019), Digitales Zentralbankgeld als neues Instrument der Geldpolitik, Wirtschaftsdienst, 99(5), 340-347, https:// www.wirtschaftsdienst.eu/inhalt/jahr/2019/heft/5/beitrag/digitaleszentralbankgeld-als-neues-instrument-der-geldpolitik.html.

Kumhof, M. und C. Noone (2018), Central Bank Digital Currencies - Design Principles and Balance Sheet Implications, Bank of England Staff Working Paper, 725.

O.V. (2019), Geld für die Heimat, Handelsblatt, Ausgabe vom 26.28.7.2019, $32 \mathrm{f}$

O.V. (2020), Bundesbank-Präsident Weidmann warnt vor Einführung eines digitalen Euros, Handelsblatt, https://www.handelsblatt.com/ finanzen/geldpolitik/geldpolitik-bundesbank-praesident-weidmannwarnt-vor-einfuehrung-eines-digitalen-euros/25362418.html (18. März 2020).

Riksbank (2020), The Riksbank's e-Krona Pilot, https://www.riksbank se/en-gb/press-and-published/notices-and-press-releases/notices/2020/the-riksbank-to-test-technical-solution-for-the-e-krona/ (20. Februar 2020)

Title: Central Bank Digital Currencies: Benefits, Risks and the Role of Blockchain Technology

Abstract: Blockchain technology can be the basis for future currencies. Numerous central banks are experimenting with this distributed ledger technology to map currencies on a blockchain basis in the future (central bank digital currencies, CBDCs). In this article, we explore motives for the introduction of CBDCs. The main motives are higher financial stability, higher security and efficiency of payment systems and a higher degree of automation of business processes. Furthermore, the risks of CBDCs are presented and the current state of development at the European Central Bank (ECB) is discussed.

JEL Classification: E24, E44, G21 